\title{
The Hardware Design of Natural Rubber Plantation Temperature and Humidity Collecting System Based On ZigBee
}

\author{
Jin Zhi-yang ${ }^{1,2}$, Li Zhong-xing ${ }^{1, *}$, Jiang Hong $^{3}, \mathrm{Xu} \mathrm{Xing}^{1}$ and Li Mei ${ }^{2}$ \\ ${ }^{1}$ College of Automotive and Traffic Engineering of Jiangsu University, Zhenjiang, Jiangsu, China \\ ${ }^{2}$ College of Electrical and Mechanical engineering of Hainan University, Haikou, Hainan, China \\ ${ }^{3}$ College of Mechanical Engineering of Jiangsu University, Zhenjiang, Jiangsu, China
}

\begin{abstract}
Natural rubber is widely used in industry, national defense, traffic, medical and daily life, but during its growth process, its productivity is influenced by the soil, temperature and humidity badly. With the progress of science and technology, how to use the information system to informationize and intelligentize the tropical agriculture is an essential research content as well as to transfer the agriculture into a modern one. Zigbee has the advantages of low power consumption, flexible net combination, quickly setting and reliable working. This article has minutely designed the hardware structure of the natural monitoring system in which Zigbee wireless sensor network is used to collect and monitor the information of rubber plantation environment's temperature and humidity. A system hardware circuit is also given in this article to meet the requirements of natural rubber monitoring.
\end{abstract}

Keywords: Rubber plantation, Temperature and Humidity collecting, Wireless sensor network, Zigbee technology.

\section{INTRODUCTION}

Rubber tree native to the Brazilian Amazon, is mostly planted in Brazil and secondly in Peru, Colombia, Ecuador, Guyana, Venezuela and Bolivia. Now it is found in over 40 nations or regions of Asia, Africa, Oceania and Latin America. The countries now with large areas in the world are Indonesia, Thailand, Malaysia, China, India, Vietnam, Nigeria, Brazil, Sri Lanka, Liberia, etc. More than 30 countries or regions have planted rubber trees while rubber is mostly produced in Southeast Asia [1]. In China, natural rubber plantation areas are located in Hainan, Guanggong, Guangxi, Fujian and Yunnan, which are mostly between two 10degree north latitude lines where the north boundary is Yingjiang, Yunnan, in the zone between 24 degrees 24 minutes and 25 degrees 20 minutes of north latitude. Besides, Taiwan is also available to plant rubber trees while Hainan is the main rubber planting area [2].

Rubber tree grows well when the temperature and humidity are high as well as the soil is fertile. It demands an average annual temperature of 26 to 27 degrees Celsius and it still produces rubber when it is 20 to 30 degrees Celsius. However it cannot bear the low temperature and gets hurt when it is below 5 degrees Celsius. The annual average precipitation is required to be between $1150 \mathrm{~mm}$ to $2500 \mathrm{~mm}$, but the altitude must be relatively high. Rubber tree grows better in thick, fertile, wet and well-drained sandy acidic soil.

*Address correspondence to this author at the College of Automotive and Traffic Engineering of Jiangsu University, Zhenjiang, Jiangsu, China;

Tel: 13976126683; E-mail: jinzhiyang94@163.com
Its roots are short and the branches are vulnerable thus it cannot defend strong winds or low temperature [3].

Also, rubber tree demands a wet atmosphere of $80 \%$ to be alive and when getting a 6-hour sunshine everyday it grows better. Known the demands of rubber trees, peasants need to control the temperature and humidity manually based on the weather forecast after growing the trees. It plantation costs enormous human resources. Meanwhile the diseases caused by a wrong temperature or humidity cannot be foreseen. In order to realize the modern forestry management, the distribution of the temperature and humidity in the whole woods should be available to collect to minutely control the irrigation systems, insulation systems, etc., then providing the best surroundings to guarantee a high quality, liberating the human resources, reducing the peasants' burdens and improving the progress of modernized technology. The occurring of the wireless sensor network based on ZigBee [4] raised an efficient and scientific solution to collect the temperature and humidity of the rubber woods and provided a theoretical basis to realize the modern forestry management.

\section{BRIEF INTRODUCTION OF ZIGBEE}

ZigBee technology was firstly introduced by Mitsubishi, Samsung, Motorola, Philip, etc. And at present, more than 100 enterprises have taken part in which include some famous enterprises in cyber, electronic or communication fields. Zigbee is built based on IEEE802.15.4 standard. The physical layer and data chaining in the technical protocol use IEEE802.15.4 standard while ZigBee Union makes the network layer and application layer. IEEE802.15.4 only 
standardizes the physical layer (PHY) and medium access layer (MAC), but the integral ZigBee protocol stack [5] is made of high-level application, specification, application convergence layer, network layer, data chaining layer and physical layer. The application layer provides multiple application interface routines to meet the users' demand. Users are able to formulate relative software [6].

\subsection{The Advantages of ZigBee System}

Compared with the other wireless networks, ZigBee's advantages are shown below [7]:

1) Low power consumption: The transmission power of the Zigbee module is only $1 \mathrm{mw}$. Mere two AA batteries can support a ZigBee facility to work for a half to two years.

2) Low overhead: Every ZigBee core costs less than $\$ 2$ and ZigBee protocol is free. The protocol is so simple that it contains only 26 pieces of management primitives.

3) Low speed: ZigBee works at a speed of 20 to 250 kbps, dividedly providing a $250 \mathrm{kbps}$ speed $(2.4 \mathrm{GHz}$ Unlicensed Bands Worldwide), a $40 \mathrm{kbps}$ speed (915 MHz U.S. unlicensed band) and a $20 \mathrm{kbps}$ speed ( $868 \mathrm{MHz}$ European unlicensed band) of raw data throughput to meet the low speed transportation demand.

4) Short distance: The transport range is between 10 to $100 \mathrm{~m}$. When increased the RF transmit power, the distance would be up to 1 to $3 \mathrm{~km}$. The 'distance' is the length between two close nodes. If the signals are transported one by one, the transport distance would be longer.

5) Low delay: ZigBee has a short response time. It can start working from the standby within 15 minutes and connects to the network in 30 minutes. It is efficient enough to reduce the duty cycle and save more power.

6) Big network capacity: A ZigBee network contains 255 nodes, which can be extended to 65535 . The network topologies include three types: star topology, mesh topology and mixed type which is convenient to join, manage and set networks.

7) Reliable and safe data transportation: Direct sequence spread spectrum (DSSS) is used on the physical layer to guarantee the reliability. ZigBee provides a function to check data integrity based on cyclic redundancy check. Authentication and certification are accessible as it uses AES-128 encryption algorithm and security mechanisms are ready for the applications.

\subsection{The Structure of ZigBee System}

It is called a wireless sensor network since $\mathrm{ZigBee}$ is mixed with sensors. A typical wireless sensor network is made of three kinds of nodes: normal nodes, sink nodes and gateway nodes [8]. Normal and sink nodes aim to collect data and realize multi-hop relay transmission. Gateway nodes are used to realize the transformation between current and wired signals to join the Internet and LAN. In the ZigBee network, normal nodes are completed by RFD while sink and gateway nodes are finished by FFD.

\section{(1) FFD: Full Function Device}

FFD is able to coordinate the network and form a network, as well as connect the other FFDs or RFDs. FFD also has a function of controlling and two-way information transmission. FFD comes with all 802.15.4 functions and all the features specified by the standard. It has more memories and calculating capability to be an extra router or terminal. Such devices can be in a tree network upstream or mesh network center and they may be arranged in a honeycomb hexagonal center in the rubber woods for region-wide coverage and optimize network transfer rate.

\section{(2) RFD: Reduced Function Device}

RFD can only send information to FFD or to receive information from an FFD. It comes with limited functions to control the cost and complexity thus ZigBee achieves natural cost savings simply. Since RFD dispenses with memory and other circuit, the ZigBee parts' cost has been reduced. As RFD is usually used as a terminal device in the network and simple 8-bit processor and a small protocol stack also help to reduce costs, such devices can be full-range efficient features in rubber woods.

Generally, gateway nodes, sink nodes and normal nodes are separately delivered to coordinator, router and terminal [9].

Coordinator: Coordinator, also called as Gateway, is responsible for starting the whole network. It is also the first device of the network. Coordinator chooses a channel, and a network ID (also called the PAN ID, i.e. a Personal Area Network ID), and subsequently starts the entire network. The coordinator can also be used to assist in the establishment of security in the network layer and application layer binding.

N.B.: Coordinator's role is mainly involved in the startup and configuration of network. Once these are completed, the coordinator of the work vanishes like a router (or disappears). Due to the distribution characteristics of the ZigBee network itself, the entire following operation of the network is no longer dependent on the coordination existence.

Router: Router is also called repeater. The main functions of the router: allows other devices to join the network, multihop routing, and assists in the communication of its own sub-terminal of the battery-powered devices. Typically, the router has been in active, so it must use the main power supply. However, when using such a network mode of tree mass, it allows the router to work cyclically thus batteries can be used to supply.

Terminal Device (End Device): The terminal device is not responsible for the specific maintenance of the network structure. It can be waken or just can be put on standby mode, so it is called a battery-powered device. Typically, the terminal device requires relatively small storage space (specially RAM), so it is the cheapest device in the entire network.

ZigBee network can contain up to 65,000 wireless data transmission node. Between each network node, the communication distance could be 75 meters to a few kilometers. 


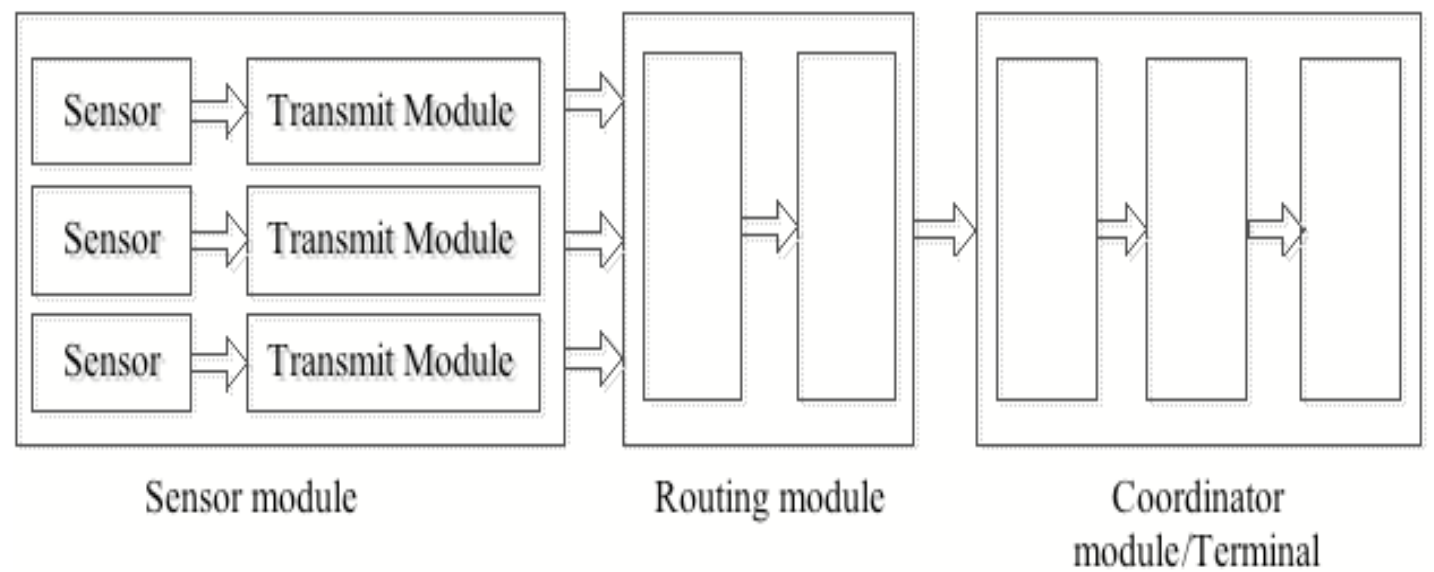

Fig. (1). Figure of design concept.

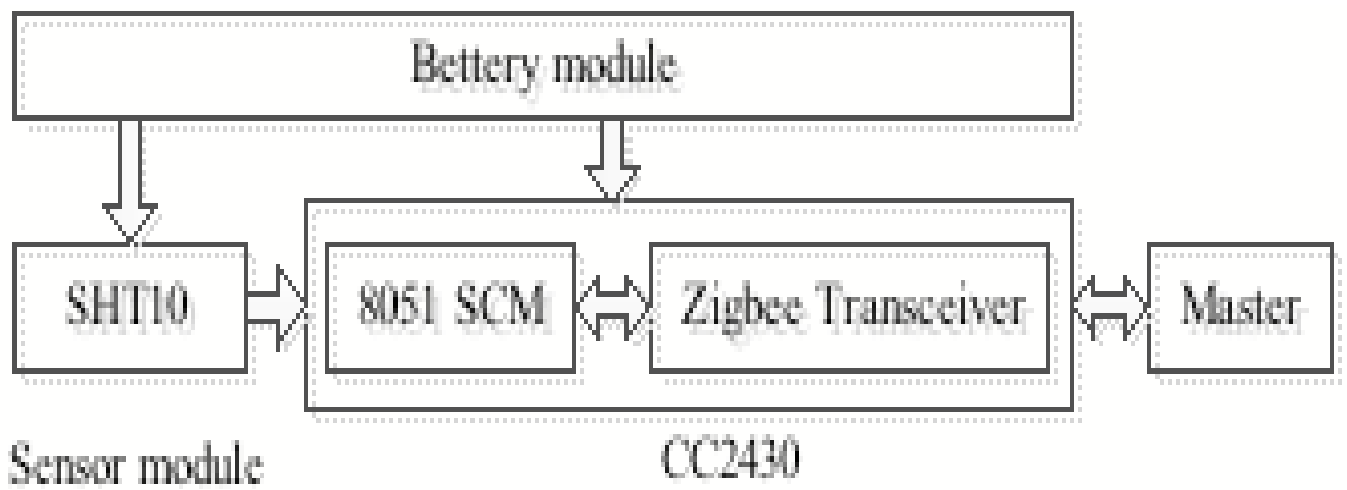

Fig. (2). Schematic Diagram of Sensor Node Structure.

Data in the wireless network coverage in each node can communicate with each other and then is eventually get transmitted to the main control center. The entire ZigBee network can also connect through the gateway with other networks such as WLAN, GSM or Internet network and finally remote monitoring of the rubber woods of environmental information can be realized.

\section{THE DESIGN CONCEPT OF THE SYSTEM}

The system design is actually the design of each node. This very system is a wireless system made of several temperature and humidity sensors, routers and coordinators. Wireless communication protocols between the various devices use ZigBee protocol. Sensor nodes as terminal device are responsible for real-time temperature and humidity data collecting within the rubber plantation, then transmitting that data to the coordinators in the method of multi-hop and finally through the serial port RS232 to connect host PCs [10] in order to collect information from sensors. Then the host PC software commands the sensors nodes to realize bothway communication and remote command, and finally realizes the wireless remote humidity monitoring and control, as shown in Fig. (1).

\section{THE DESIGN OF SENSOR NODE}

The wireless sensor node is mainly constituted by the most inexpensive terminal device. Besides, it also includes some of the individual sensor nodes and routing node router. Its main function being distributed in the rubber woods is to monitor the temperature and humidity of the area, real-time collection of temperature and humidity parameters and transmission of the data to the superior routing nodes or gateway nodes. Wireless sensor node includes processor module, wireless transmit-receive module, sensor module and battery module. The structure is given in Fig. (2).

The basis of the entire module is built on the power module. The power module is divided into two categories. The field work equipment uses two AA batteries to provide energy to the other three modules node, as shown in Fig. (3); and indoor or close to the stable power supply equipment by a 5V DC power supply is given in Fig. (4). In order to simplify the user to select, and make the devices get access to power for becoming freely interchangeable, all equipments contain both these two power circuit.

Sensor nodes in the rubber woods detect temperature and humidity data through temperature and humidity sensors SHT10, and then transfer the data to the CC2430 signal processing and send the signals out. CC2430 is a product launched by TI's acquisition of Chipcon. It is the world's first true sense of the system-on-chip of Zigbee solution, which is a product truly meeting the IEEE802.15.4 and Zigbee standards. CC2430, used in this system, integrates an enhanced 8051- microcontroller core with 8-bit single-cycle is used to operate and store the temperature and humility information. The wireless transmit-receive module, made up with the 


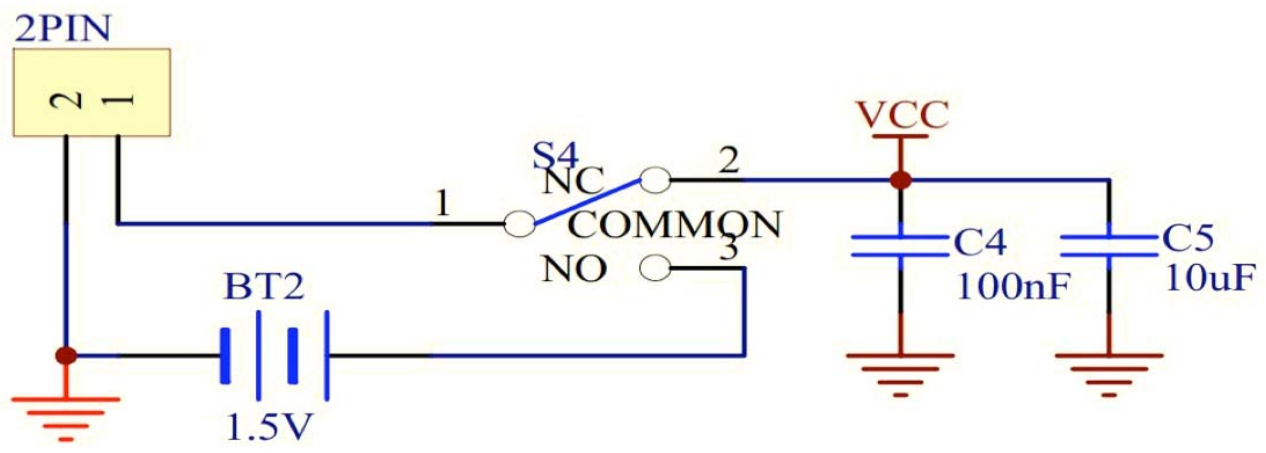

Fig. (3). Power Supply Circuit with Two-1.5V Batteries.

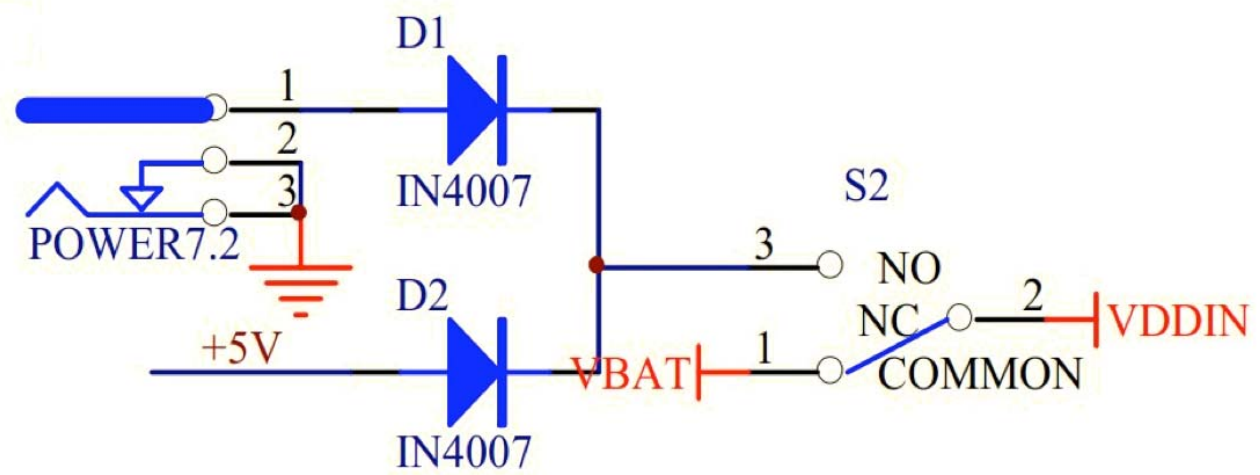

Fig. (4). DC 5V Power Supply Circuit.

transmitting terminal and receiving terminal, embedded Zigbee wireless RF module, is made to communicate with the other nodes. Part of the work of the entire wireless transceiver module $2.4 \mathrm{~Hz}$ band and It uses low-voltage power supply and has the advantages of low power and excellent $\mathrm{RF}$ performance. The CC2430 chip has high receiver sensitivity and robustness is sufficient to be operating in the field of environment. It has $8 \mathrm{~kb}$ SRAM inside and the capability of holding data in a variety of power supply. In the Powerdown mode, the current consumption of the chip is only 0.9 $\mathrm{uA}$ and external interrupt or Real Time Clock (RTS) are able to wake up the system; While in suspend mode, the current consumption of the chip is less than $0.6 \mathrm{uA}$ and external interrupt is able to wake up the system. While it guarantees a sufficiently small duty cycle, as much as possible to increase its field endurance, reduce equipment maintenance cycle, and enhance the usefulness of the device.

Find CC2430 chip Handbook to know the definition of each pin as Fig. (5).

The $\mathrm{P}_{0}$ port is data input, $\mathrm{P} 1$ port is data output, $\mathrm{P} 2$ port is a hybrid interface reserved for more procedures and functional development. The details of the specific role of each pin will be introduced below.

The sensor module, made of SHT10 digital temperature and humidity sensor, is used to collect relative information in those wireless covered areas. SHT10 sensor is a temperature and humidity sensor containing a calibrated digital signal output. At $25^{\circ} \mathrm{C}$, the humility and temperature measuring precision is $\pm 4.5(\% \mathrm{RH})$ and $\pm 0.5\left({ }^{\circ} \mathrm{C}\right)$. The product has excellent quality, fast response, anti-interference ability, cost and other advantages. The sensor includes a capacitive polymer humidity measuring device and energy gap tem- perature measurement devices connected seamlessly with a 14-bit A / D converter and a serial interface circuit on the same chip. Similarly, we refer to the relevant chip Handbook and get a pin circuit diagram as Fig. (6).

The DATA pin and SCK pin of sensor SHT10 are separately connected with $\mathrm{P} 0.5$ pin and $\mathrm{P} 0.4$ pin of CC2430. CC2430 communicates with sensor through I2C protocol to gain the real-time temperature and humidity. In order to compensate for the non-linearity of the humidity sensor to obtain accurate data, Formula 1 shown below is used to fix the data [11]:

$$
\begin{aligned}
& R H_{\text {linear }}=c_{1}+c_{2} \cdot S O_{R H}+c_{3} \cdot S O_{R H}^{2} \\
& \text { In the formula, } \mathrm{C}_{1}=-2.0468, \mathrm{C}_{2}=0.0367 \text {, and } \\
& \mathrm{C}_{3}=-1.5955 \mathrm{E}-6 .
\end{aligned}
$$

The formula 2 is used to translate the data output into the value of temperature:

$T=d_{1}+d_{2} \cdot S O_{T}$

In the formula, $\mathrm{d}_{1}=-39.6, \mathrm{~d}_{2}=0.01$.

\section{THE DESIGN OF ROUTING NODE}

The structure of routing node is generally the same as that of wireless sensor node. In order to reduce the overhead, the sensor module can be simplified. However, in the CC2430 chip of the router node, the value of nwkRouting is different from that of the sensor node.

$\mathrm{Cm}$ is defined as the maximum of child nodes, $\mathrm{Rm}$ as the maximum of routing nodes and Lm as the maximum net- 


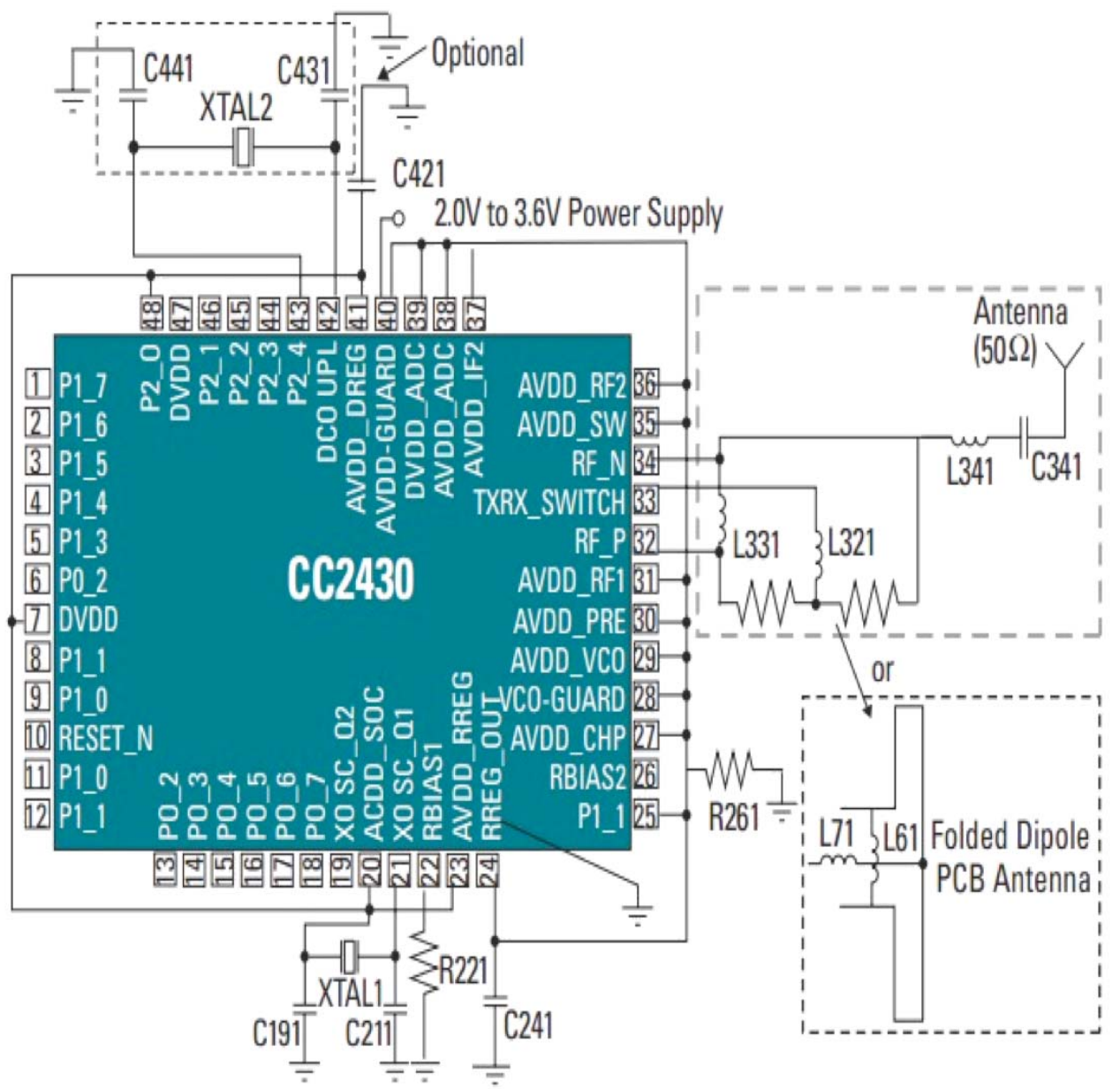

Fig. (5). CC2430 Chip.

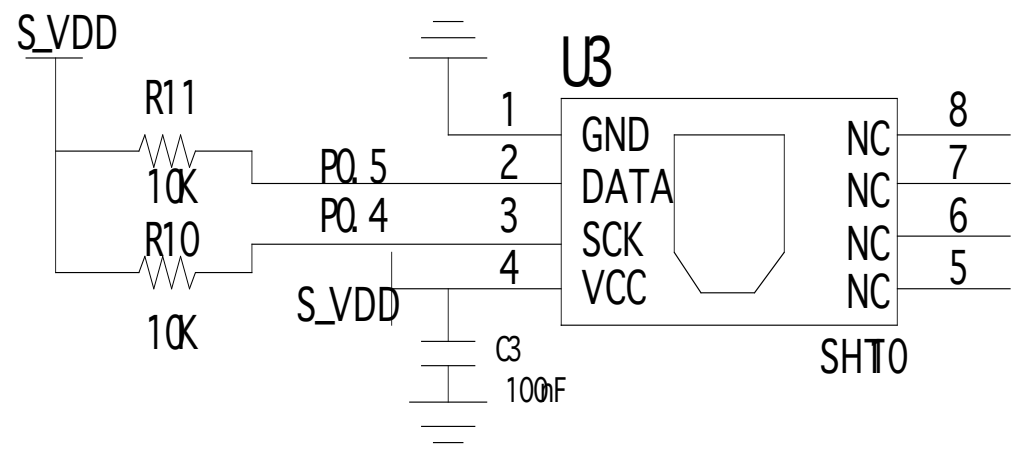

Fig. (6). SHT10 Sensor Circuit.

work depth. Therefore, if the routing node with a d-deepnetwork can deliver an address space Cskip(d), delivered to the k-th address Ak and the n-th terminal address An meets the formula below [12]:

$$
\begin{aligned}
& \mathrm{C}_{\text {skip }}(d)=\left\{\begin{array}{cc}
1+C_{m} \cdot\left(L_{m}-d-1\right) & \cdots \cdots \cdots \cdots\left(R_{m}=1\right) \\
\frac{1+C_{m}-R_{m}-C_{m} R_{m}^{L_{m}-d-1}}{1-R_{m}} & \cdots \cdots \cdots \cdots\left(R_{m} \neq 1\right)
\end{array}\right. \\
& A_{k}=A_{\text {father }}+C_{\text {skip }}(d) \cdot(k-1)+1 \\
& A_{n}=A_{\text {father }}+C_{\text {skip }}(d) \cdot R_{m}+n
\end{aligned}
$$

$\mathrm{A}_{\text {father }}$ in the formula means the delivered father address of the network.

In order to increase the transmission distance of the route, you can appropriately choose high power RF transceiver module configured to expand the antenna. In the router module, in order to reduce the volume of the products we directly embed CC2430 chip in the RF module, given as Fig. (7). Then we lead $\mathrm{P}_{0.0}$ to $\mathrm{P}_{0.7}$ pin to capture downstream node data and $\mathrm{P}_{1.2}$ to $\mathrm{P}_{1.7}$ to send data to the parent node. And if the routing module is integrated in sensor module, $\mathrm{P}_{2.0}$ to $\mathrm{P}_{2.2}$ pin is reserved for the routing module data acquisition and data communication. 


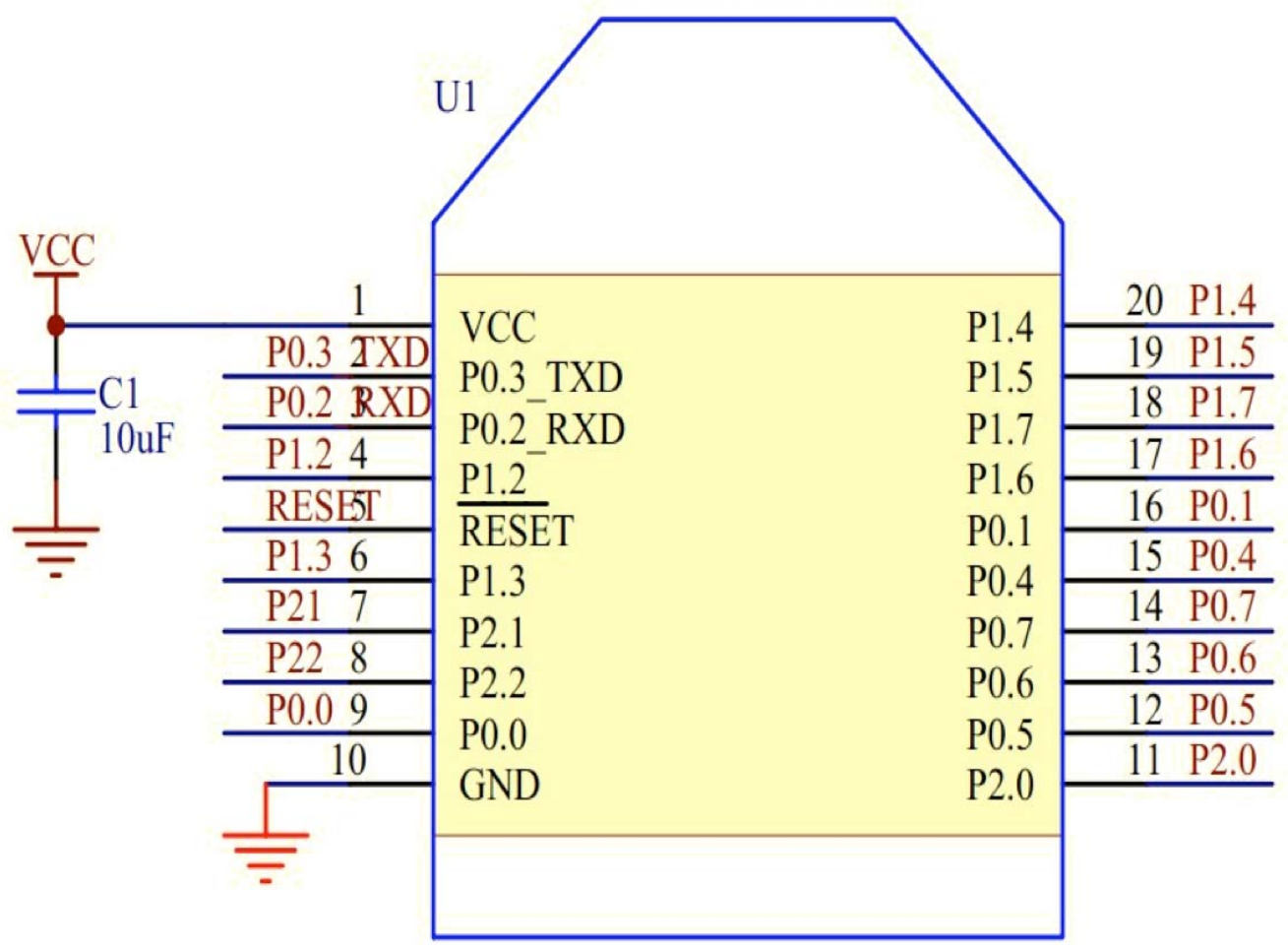

Fig. (7). RF modules.

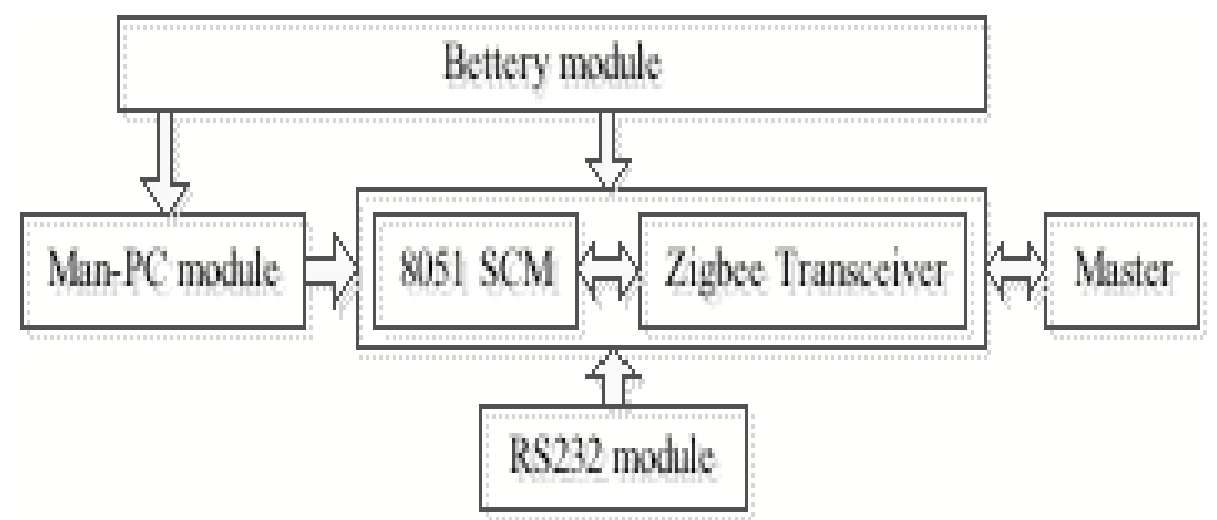

Fig. (8). Schematic Diagram of Coordinator Node.

\section{THE DESIGN OF THE COORDINATOR NODE}

Coordinator node is made of the processor module, wireless transceiver module, RS232 communication module, human-computer interaction module and power module. The design of the processor module, wireless transceiver module and power module is the same as that of the wireless sensor node. The actual schematic diagram is shown below in Fig. (8):

RS232 communication module is used on SoC chip to communicate with PC serial ports. The data transmission between the Zigbee coordinator CC2430 and C8051F320 chip is a serial transmission. The most common way is to use the RS-232 interface, realized by the UART dedicated chip circuit [13]. In the system, in order to achieve the microcontroller UART interface with RS232 interface of the PC connection, we need to use a SP3223E chip as RS232 interface chip to do level conversion. Between two different logic levels, they can not directly communicate.

EIA RS-232C uses positive and negative voltage to represent a logic state, whlie the CC2430 control chip uses TTL high and low logic state. The two kinds of standards are not compatible. Therefore a level switch is necessary [14]. Based on the standard EIA-RS-232C, Txd and Rxd are stipulated as logic 1 MARK=-3V -15V and logic 2 SPACE $=+3 \mathrm{~V} \sim+15 \mathrm{~V}$. The SP3223E supply voltage is compatible in the range of $3 \mathrm{~V}-5 \mathrm{~V}$, with a power consumption of $0.3 \mathrm{~mA}$ and an external $40.1 \mathrm{uF}$ capacitor. The connection circuit between UART and the SP3223E is given as Fig. (9). In this system, 9 pins are not all used as only 3 are available. Pin 5 is used to connect tot he GND, Pin 3 to connect SP 3223E's Pin7 as a reciever, Pin 2 to connect SP3223E's Pin8 as a transmitter. The Pin 9 and 10 are dividedly connected to the microcontroller C8051F320 chip's TxD, RxD. 


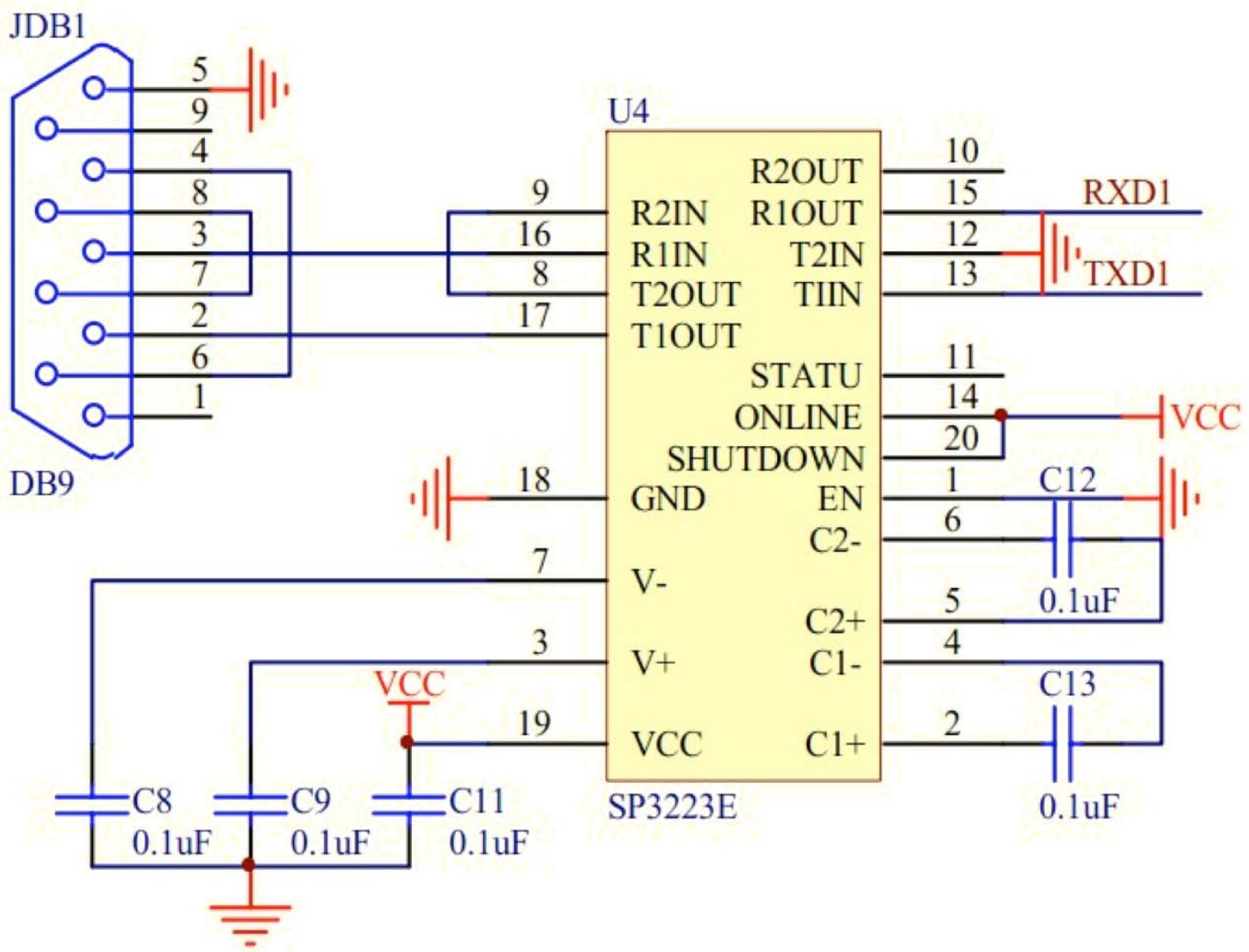

Fig. (9). Diagram of Serial Circuit.

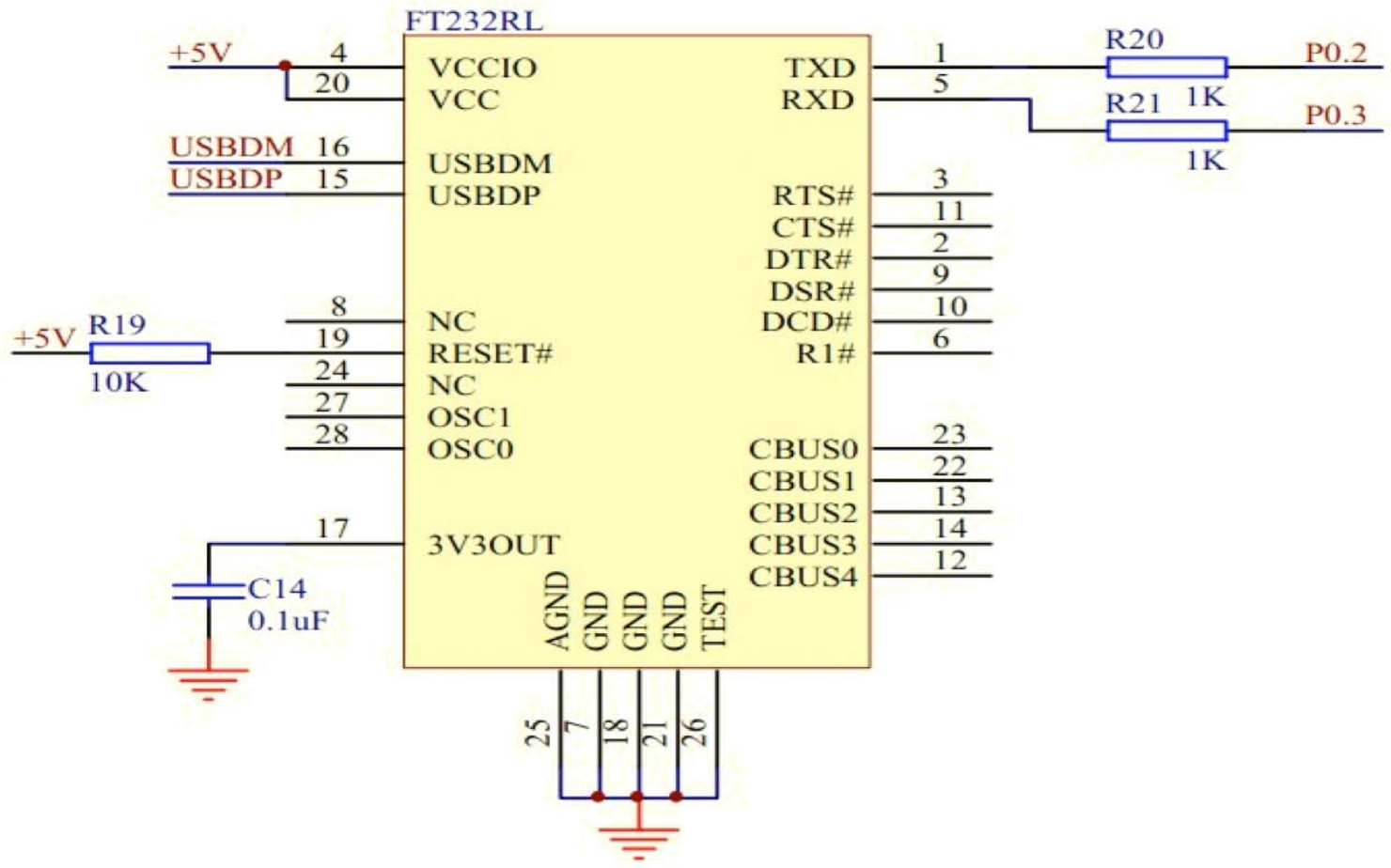

Fig. (10). USB-to-Serial Chip.

At the same time, considering that many host PCs are no longer integrated with RS interface but USB interface. We added FT232RL chip into the coordinator. FT232RL is a FTDI USB-UART bridging product. It has an onboard
EEPROM and the master clock generator, 3.3V LDO regulator, reset generator and USB termination resistors, given as Fig. (10). It can be realized to convert USB to serial UART interface can also be converted to synchronous, asynchro- 


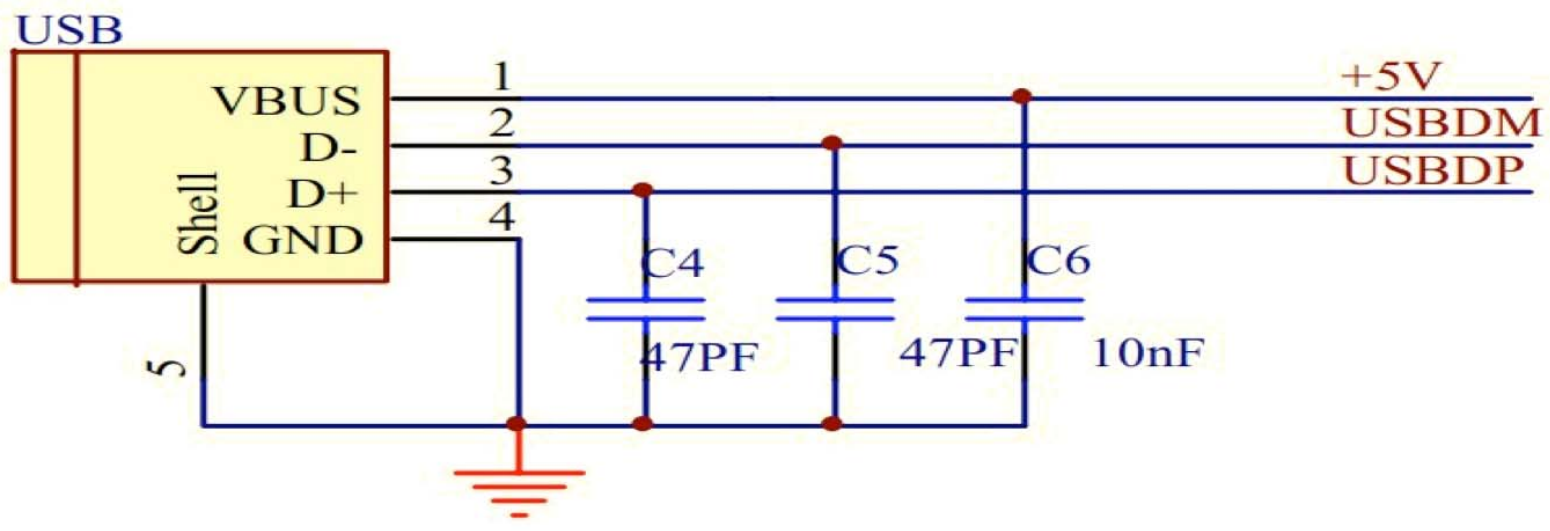

Fig. (11). USB Circuit.

nous interface mode BIT-BANG as Fig. (11). The USB to RS232 devices, which FT232RL provided, rolled both RS and USB communication protocol's advantages into one. USB interface's plug-and-play and hot-swap capabilities provide a very easy-to-use environment to RS232 devices and RS232 has the characteristics of long-distance transmission and anti-jamming performance that has given the USB port function of the long-distance transmission with a speed of 3 Mbps.

Human-computer interaction module includes keyboard input module, LED indication module and LCD display module. However, in this design, the PC host computer will be master program, so only the LCD display module is left in human-computer interaction module, with Univision dot matrix LCD UG12864, used to display the temperature and humidity data from each route summarization of all nodes.

\section{CONCLUSION}

The hardware design of natural rubber plantation temperature and humidity collecting system in this article is based on ZigBee wireless networking platform, which is a wireless mesh network composed of wireless sensor nodes with temperature and humidity sensors, a number of wireless nodes with routing functionality and network coordinator. Wireless communication protocols between the various devices use the ZigBee protocol. Sensor node is used to collect the real-time data of the temperature and humidity of rubber woods. Then data is transmitted through the wireless network to the router nodes, router nodes according to the routing algorithm select the most suitable communication path in order to collect the value of temperature and humility from each node. Commands are sent through host PC software to each sensor node to realize the both-side communication and eventually to remote monitor the temperature and humility of the rubber woods. It is a typical example of ad hoc networks using wireless sensing system effectiveness and advantages in the field of natural rubber plantation agricultural equipment monitoring. The system created by the ZigBee network platform and associated online monitoring system will be more flexible in temperature and humidity acquisition system to play its utility.

\section{CONFLICT OF INTEREST}

The authors confirm that this article content has no conflicts of interest.

\section{ACKNOWLEDGEMENTS}

Financial supports from: (1)Acquisition System Design of Temperature and Humidity Based on Wireless Sensor Network(rdzwkfjj006), Hainan Tropical Crops Research of Information Technology Open Fund of Key Laboratory Project ; 2Logistics Development Mode Research of Yangpu Port(hd09xm89), Hainan University 2009 Research Project ; (3)Tourism Logistics System Coordination Mechanism and Modeling Analysis Research Based on System Dynamics(CXZZ11_0580), Jiangsu 2011 University Postgraduate Research and Innovation Project.

\section{REFERENCES}

[1] W. Zhi-xiang, et al., "The carbon and total nitrogen characteristics of different age rubber plantation soil in danzhou of hainan", J. Eco. Environ. Sci., vol. 18, no. 4, pp. 1484-1491, 2009.

[2] W. Zhi-xiang, X. et al. "The initial observations of rubber planation ecosystem dry season microclimate characteristics and flux", J. Trop. Crops, vol. 12, pp. 2081-2090, 2010.

[3] W. Zhi-xiang, et al., "The distribution study of rubber forest flux footprint and source area in hainan", J. Trop. Crops, vol. 1, pp. 42 $50,2012$.

[4] L.Wen-zhong, et al., "ZigBee wireless network technology's introduction and actual combat", Beijing: Beijing University of Aeronautics and Astronautics Publishing House, 2007, pp.1-12.

[5] L. Zhi-an, The principle and application development of ZigBee network. Beijing University of Aeronautics and Astronautics Publishing House: Beijing, 2008, pp.18-32.

[6] C. D. G. Zhi Information Technology Co., Ltd, The introductions of ZigBee wireless sensor network application development system WSN-CC2430 Enterprise ed, 2010.

[7] W. Yan, S. Guohua, "Zigbee wireless sensor network technology and application", Ship Electron. Eng., vol. 28, no. 10 pp. 32-33, 2008.

[8] L. Quan, The Research on ZigBee Wireless Sensor Network and the Application in Hazard Monitoring System. Lanzhou University of Science \& Technology: Lanzhou China, 2010.

[9] W. Fengbo, Q. Yunxiao. J. Northwest University (Natural Science Edition), vol. 38, no. 5, pp. 731-734, 2008.

[10] L. Hua, L. Wuyi, X. Hongyun, "Programmable Controller \& Factory Automation”, pp. 53-61, 2010. 
[11] Dalian North Measurement and Control Engineering Co., Ltd. Humidity \& Temperature Sensor SHT1x/SHT7x[Z]. Dalian: Dalian High-tech Zone Students Street No. 2 -3-1-2(2007).

[12] H. Lingling, "An improved cluster tree routing algorithm in zigbee networks”, Chin. J. Sens Actuator, pp. 1303-1307, Sept. 2010.
[13] Z. Yi, The topology design and realization of wireless sensor network based on zigbee technology. Suzhou University: Suzhou, 2009.

[14] S. Cai-yun, L. Shi-zhong, L. Li-li, "The equipment monitoring system design based on zigbee technology", Water Electric Power Sci., vol. 28, no. 11, pp.125-127, 2010.

Received: October 09, 2013

Revised: December 20, 2013

Accepted: December 24, 2013

(c) Zhi-yang et al.; Licensee Bentham Open.

This is an open access article licensed under the terms of the Creative Commons Attribution Non-Commercial License (http://creativecommons.org/licenses/by-nc/3.0/) which permits unrestricted, non-commercial use, distribution and reproduction in any medium, provided the work is properly cited. 